\title{
ЭНТРОПИЙНО-ИНФОРМАЦИОННЫЙ АНАЛИЗ САМООРГАНИЗАЦИИ И ЭФФЕКТА ФУНКЦИОНИРОВАНИЯ ИНДУСТРИАЛЬНОЙ ЭКОНОМИЧЕСКОЙ СИСТЕМЫ
}

\author{
C. В. Чупров \\ Байкальский государственный университет, г. Иркутск, Российская Федерация
}

\section{Информация о статье}

Дата поступления

15 мая, 2017 г.

Дата принятия к печати

5 июня, 2017 г.

Дата онлайн-размещения 15 сентября, 2017 г.

\section{Ключевые слова}

Информация; порядок; самоорганизация; синергетика; хаос; энтропия; эфрфект

\begin{abstract}
Аннотация
Актуальность решения обсуждаемой проблемы обусловлена возрастающей динамичностью экономических систем и их окружения, что вызывает в них нелинейные процессы с присущими им особенностями. Привлечение концепций и инструментария теории информации и синергетики позволяет лучше понять роль и характер воздействия возмущений и энтропии на функционирование и самоорганизацию индустриальных экономических систем. Эволюционирующая в настоящее время экономика по характеру поведения является нестационарной, что служит причинами ее хаотизации и метаморфоз в возмущенном окружении. В статье излагаются теоретические вопросы взаимовлияния энтропии и информации на хаотизацию и организованность экономических систем, с позиций энтропийного и информационного подходов раскрываются природа и условие самоорганизации в индустриальных экономических системах, проводится анализ модификации модели В. А. Трапезникова, в которой формализуется связь показателей энтропии, управляющей информации и эффекта функционирования экономической системы, а также влияния показателя порядка поведения индустриальных экономических систем на эфрфект их деятельности. Перспективным направлением дальнейших исследований видится изучение динамических свойств самоорганизации и развития индустриальных экономических систем с позиции устойчивости их функционирования.
\end{abstract}

\section{ENTROPY-INFORMATION ANALYSIS OF SELF-ORGANIZATION AND OF THE EFFECT OF FUNCTIONING OF AN INDUSTRIAL ECONOMIC SYSTEM}

\author{
Sergey V. Chuprov \\ Baikal State University, Irkutsk, Russian Federation
}

Article info

Received

May 15, 2017

Accepted

June 5, 2017

Available online

September 15, 2017

\section{Keywords}

Information; order; selforganization; synergetics; chaos; entropy; effect

\begin{abstract}
The problem under discussion is important today because of the increasing dynamism of economic systems and their environment; that causes non-linear processes (with their intrinsic particularities) in these systems. Attracting concepts and tools of information theory and synergetics allows us a better understanding of the role and nature of the impact of disturbance and entropy on functioning and self-organization of industrial economic systems. The developing nowadays economy in its behavioral character is non-stationary. These are the causes of its chaotization and metamorphosis in perturbed environment. The article sets out theoretical matters of interinfluence of entropy and information on chaotization and organization of economic systems. Basing on the entropy and informational approaches, the author also analyses the nature
\end{abstract}


and requirement of self-organization in industrial economic systems. The author analyses the modification of the V. Trapeznikov model, which formalizes the connection of indices of entropy, control information and the effect of functioning of the economic system. He also analyses the influence of the order parameter on the behaviour of industrial economic systems. The author sees the study of dynamic properties of selforganization and the development of industrial economic systems from the standpoint of the stability of their functioning as a promising line of further research.

Воззрения и методологический арсенал современной информационной концепции формировались «в сплаве» бурно развивающихся научных течений, восходящих прежде всего к термодинамике и статистической физике, а в последующем и кибернетике. Статистическое толкование и энтропийную интерпретацию поведения систем питали идеи фундаментальных исследований Р. Клаузиуса [1], Л. Больцмана [2], Дж. Гиббса [3], М. Смолуховского [1]. Введя в научный обиход понятие энтропии, Р. Клаузиус установил, что в замкнутой системе она остается либо неизменной (обратимые процессы), либо возрастает (необратимые процессы). Придавая энтропии вероятностную трактовку, Л. Больцман доказал, что «всякая замкнутая система тел стремится к определенному конечному состоянию, для которого энтропия будет максимум» [2, с. 176-177].

Согласно учению Дж. Гиббса, добиться уменьшения энтропии в системе возможно при подводе к ней энергии, которая противостоит разрастанию хаоса в системе [3]. Между тем его понимание энтропии привело к аргументации ее влияния на упорядоченность системы. Один из создателей квантовой механики Э. Шредингер проницательно заметил, что взятая с отрицательным знаком энтропия есть сама по себе мера упорядоченности, поэтому наращивание отрицательной энтропии (называемой негэнтропией) средство обуздания и ослабления хаоса, распространение которого может довести до деградации и разрушения системы [4].

Зависимость между энтропией, организованностью и количеством информации в системе была обстоятельно исследована классиками кибернетики, и в нее наряду с некоторыми другими профильными областями знаний стала входить и теория информации [5, с. 35], образуя ее раздел [6, с. 396]. Н. Винер полагал, что «как количество информации в системе есть мера организованности системы... точно так же энтропия системы есть мера дезорганизованности системы; одно равно другому, взятому с обратным знаком» $[7$, с. 56]. В дальнейшем эту новаторскую идею успешно развивали в своих трудах Л. фоон Берталанфи [8], С. Бир [9], Л. Бриллюэн [10], К. Шеннон [11], У. Эшби [12] и др.

Полная острых институциональных противоречий и динамизма социально-экономических процессов, переживаемая эпоха рыночных преобразований сопровождается нарастающим влиянием на деятельность хозяйственных структур потока деструктивных возмущений, способных вызвать хаотизацию поведения индустриальных экономических систем, в частности, промышленных предприятий. Вследствие этого в нестационарной среде они претерпевают качественные метаморфозы и перестройки, смешение медленных и быстрых, плавных и скачкообразных фраз, возникновение и распад витиеватых структур.

Хаотизация системы нарушает упорядоченность ее поведения, причем становятся чувствительными даже слабые возмущения, которые могут породить принципиально иной режим поведения эволюционирующей системы, ведь в контексте синергетики хаотическая система с течением времени подвергается замысловатым преобразованиям с обретением упорядоченного режима функционирования.

В поиске меры порядка в системе Г. Ферстер логично предположил, что этой цели может служить «относительная энтропия» выражение для определения избыточности [13], которое он заимствовал у основоположника теории информации К. Шеннона:

$$
R=1-\frac{H}{H_{m}},
$$

где $H$ - энтропия источника информации; $H_{m}$ - максимально возможная энтропия источника информации.

Легко увидеть, что величина показателя $R$ принадлежит интервалу от 0 до 1 , и ее граничные значения отвечают отсутствующему порядку в системе, если величина равна 0, и «совершенному» порядку, если она равна 1. В случае, когда энтропия в системе становится предельно высокой $\left(H=H_{m}\right)$, и тем самым она пребывает в максимальном беспорядке, показатель порядка в системе нулевой, т. е. 
$R=0 ;$ и, наоборот, когда энтропия $(H=0)$ и беспорядок в системе исчезают, показатель порядка максимален, т. е. $R=1$.

В теоретическом аспекте [14, с. 343] нетрудно показать, что самоорганизация эволюционирующей экономической системы становится возможной с выполнением условия

$$
H \frac{d H_{m}}{d t}>H_{m} \frac{d H}{d t}
$$

при котором беспорядок в поведении наблюдаемой системы уступает нарастающему порядку. Отсюда самоорганизация системы предполагает, что в этом случае диффреренциал энтропии $d H$ должен быть меньше дифференциала максимальной энтропии $d H_{m}\left(d H<d H_{m}\right)$, что указывает на относительное повышение порядка в системе.

Однако необходимо обратить внимание на то обстоятельство, что с позиций теории информации она представляет собой устраненную неопределенность поведения системы и численно равна уменьшению этой неопределенности. Пусть в исходном положении система находилась в хаотичном состоянии с максимальной энтропией $H_{m^{\prime}}$ тогда количество извлеченной иноормации I определится разностью

$$
I=H_{m}-H,
$$

а выражение (1) можно записать в следующем виде:

$$
R=1-\frac{H}{H_{m}}=\frac{H_{m}-H}{H_{m}}=\frac{I}{H_{m}} .
$$

При этом сохраняют силу предыдущие рассуждения относительно свойств показателя порядка $R$ : если система максимально хаотизирована, и ее энтропия остается самой высокой $\left(H=H_{m}\right)$, то по разности (2) количество извлеченной информации будет равняться нулю $(I=0)$, так же как и величина показателя порядка в системе $(R=0)$. В предельном случае, когда гипотетически становится возможным полностью вытеснить хаос и достигнуть «совершенного» порядка в системе при $H=0$, количество извлеченной информации по формуле (2) имеет наибольшее значение $I=H_{m}$ и показатель порядка в системе будет максимальным: $R=1$.

Вместе с тем, поскольку самоорганизация эволюционирующей системы подразумевает повышение порядка в ней, скорость изменения показателя $R$ должна быть положительной:

$$
\frac{d R}{d t}>0
$$

Принимая во внимание фрормулу (3), диффреренцирование ее дает следующее неравенство:

$$
\frac{d R}{d t}=\frac{H_{m} \frac{d l}{d t}-I \frac{d H_{m}}{d t}}{H^{2}{ }_{m}}>0 .
$$

Далее, учитывая, что знаменатель дроби всегда положительный $\left(H_{m}^{2}>0\right)$, находим условие выполнения выражения (4):

$$
H_{m} \frac{d l}{d t}>I \frac{d H_{m}}{d t}
$$

Таким образом, соблюдение этого соотношения означает, что в эволюционирующей экономической системе протекает процесс самоорганизации, и потому беспорядок в ее поведении уменьшается, а порядок растет. Несложный анализ выражения (5) дает возможность сорормулировать ряд присущих самоорганизации индустриальной экономической системы примечательных выводов. Обратимся к двум частным случаям постоянства величин этого неравенства.

Пусть величина максимальной энтропии системы не меняется с течением времени $\left(H_{m}=\right.$ const). Ввиду этого производная $\frac{d H_{m}}{d t}=0$ и для левой дроби неравенства (5) получим условие $H_{m} \frac{d l}{d t}>0$. Налицо закономерный и ожидаемый вывод: при фиксированной величине максимальной энтропии самоорганизация системы сопровождается насыщением ее управляющей информацией и, соответственно, уменьшением хаоса в системе и повышением порядка в ее поведении. Благодаря этому в процессе самоорганизации в эволюционирующей экономической системе появляется феномен когерентности и улучшается коллективное поведение ее подсистем (элементов), вплоть до модернизации и коренной реорганизации структуры.

В контексте теории управления полученный результат подводит теоретическое обоснование под хорошо известный из практики способ повышения эфффективности деятельности промышленных предприятий. В их экономической системе, охваченной хаосом, предотвращение разложения структуры и деградации достигается усилением управляющих воздействий посредством интенсификации потоков циркулирующей директивной и отчетной инорормации. Поэтому, несмотря на напористое влияние вредных возмущений и хаотизацию работы предприятий индустрии, удается стабилизировать и поддерживать эффрективный режим их деятельности. 
Если количество накопленной в системе управляющей информации ( $I=$ const) стабильно, то производная от нее $\frac{d l}{d t}=0$. Ввиду этого левая часть неравенства (5) также обращается в ноль, и получаем иное условие нарастания порядка в экономической системе:

$$
I \frac{d H_{m}}{d t}<0 .
$$

Очевиден и этот вывод для самоорганизации системы: поскольку количество инорормации всегда неотрицательно $(I \geq 0)$, выполнение этого неравенства означает снижение уровня максимальной энтропии $H_{m^{\prime}}$ что равносильно уменьшению хаоса и повышению порядка в системе.

Уместен анализ общего случая, когда в неравенстве (5) могут изменяться как величина максимальной энтропии $H_{m}$ в системе, так и количество информации I в ней. Речь идет о формализации универсального свойства самоорганизующихся индустриальных экономических систем, допускающего произвольную динамику показателей $H_{m} n l$.

Во-первых, неравенство (5) свободно от каких-либо ограничений, связанных с фиксированием постоянных значений энтропии и количества информации, и лишь обязывает соблюдать следующее соотношение: произведение величины максимальной энтропии $H_{m}$ в экономической системе и скорости наращивания в ней количества инфрормации (левая часть выражения) должно превышать произведение величины I и скорости изменения максимальной энтропии $\frac{d H_{m}}{d t}$ (правая часть выражения). При этом в динамике показателей снижение одних может быть компенсировано увеличением других при соблюдении условия (5).

Во-вторых, не исключается и согласованное изменение показателей. Так, для левой части неравенства (5) можно констатировать: если текущее значение максимальной энтропии $\mathrm{H}_{m}$ достаточно большое, то скорость роста количества информации $\frac{d l}{d t}>0$ может быть пониженной в той мере, какой такая динамика удовлетворяет неравенству (5). Тем самым в развитии системы находит свое выражение синергетический принцип И. Пригожина [15], когда из хаоса в ней образуется порядок, эволюционирующая система претерпевает перестройки и переходит в качественно новое состояние.

В-третьих, аналогичные рассуждения можно привести и для правой части неравенства (5). Благоприятной является ситуация, когда скорость изменения максимально возможной энтропии $\frac{d H_{m}}{d t}$ мала и стабилизируется, ввиду чего и количество информации I в системе может оставаться умеренным. В рамках синергетических постулатов уменьшение скорости $\frac{d H_{m}}{d t}$ способствует обузданию разрастания потенциальных хаотических процессов и повышению порядка в системе.

В-четвертых, не лишено оснований предположение, что в сильно динамизированной индустриальной экономической системе (когда скорость $\frac{d H_{m}}{d t}>0$ значительна) с достаточным объемом накопленной информации (I) ее самоорганизация по неравенству (5) предполагает быстрый рост количества инфрормации в системе $\frac{d l}{d t}$ с учетом величины максимальной энтропии $H_{m}$.

Заметим, что неравенство (5) можно преобразовать следующим образом:

$$
\frac{1}{H_{m}}<\frac{d l}{d t}: \frac{d H_{m}}{d t} .
$$

В соответствии с выражением (3) определим, что показатель порядка $R$ в системе меньше соотношения приращений в ней количества информации dl и максимальной энтропии $d H_{m}$ :

$$
R=\frac{l}{H_{m}}<\frac{d l}{d H_{m}} .
$$

В продолжение обсуждения энтропийно-информационной природы самоорганизации индустриальных экономических систем найдем зависимость эффректа ее фрункционирования от показателя порядка поведения системы. Как и прежде будем считать, что управление экономической системой направлено на снижение неупорядоченности ее поведения и, если величина неупорядоченности системы первоначально была равной $B_{0}$, то после введения в нее управляющей иноормации в определенном объеме / величина неупорядоченности системы становится $B\left(B<B_{0}\right)$. Исходя из принятого в теории информации статистического толкования зависимости эффректа функционирования экономической системы Э от количества поступившей в нее управляющей информации I, В. А. Трапезников $[16$, с. 51$]$ математически обосновал связь между ними формулой

$$
\ni=\ni_{\max }\left(1-e^{-\frac{1}{I_{0}}}\right),
$$

где $\ni_{\max }$ - эфффект идеально функционирующей системы (предельно возможный 
эффрект); $I_{0}$ - объем информации, характерный для данного объекта управления.

Выражение (6) формализует свойственную индустриальной экономической системе примечательную закономерность: с наращиванием в ней управляющей информации повышается организованность и эффрект ее деятельности, и наоборот. При этом экспоненциальный вид этой связи раскрывает нелинейный характер ее развития в «борьбе» между неупорядоченностью и информацией в энтропийном аспекте. Так, с помощью введения в экономическую систему количества управляющей информации $l_{y}$ снижается ее неупорядоченность $B$, и эффрект функционирования системы Э растет [16, с. 8] (рис.).

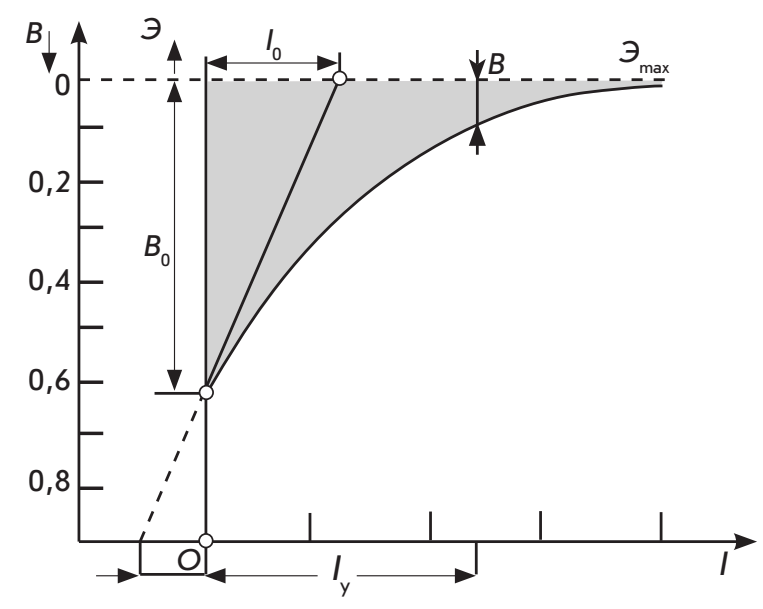

Эффект управляемого комплекса в зависимости от количества поступившей в него управляющей информации

Аргументируем зависимость эфффекта функционирования индустриальной экономической системы Э от меры порядка ее поведения, для чего введем его показатель $R$ в формулу (6). Поскольку согласно равенства (3) $I=R H_{m^{\prime}}$ с подстановкой правой части этой формулы в выражение (6) найдем искомую зависимость:

$$
\ni=\ni_{\max }\left(1-e^{-\frac{R H_{m}}{I_{0}}}\right) .
$$

Анализируя полученное выражение (7), можно сделать следующие выводы:

1. Комплексное влияние величин $H_{m^{\prime}} I_{0}$ и $R$ задает темп изменения эффректа фрункционирования индустриальной экономической системы Э.

2. Эффрект функционирования индустриальной экономической системы Э тем выше, чем больше показатель порядка ее поведения $R$. Поскольку в частном случае значения максимальной энтропии $H_{m}$ и заложенного в экономическую систему объема инфор- мации $I_{0}$ фиксируемы, эфрфект однозначно определяется показателем порядка поведения системы $R$, т. е. его увеличение обеспечивает по экспоненте рост эффекта ее работы.

3. С нарастанием хаотизации индустриальной экономической системы и ее максимальной энтропии $H_{m}$ для стабилизации и повышения эфффекта функционирования Э необходимо улучшение организованности системы посредством активного насыщения ее управляющей информацией, отмечаемого увеличением показателя порядка поведения $R$.

4. С возрастанием объема информации $I_{0}$ ужесточаются требования к положительной динамике показателя порядка поведения $R$ индустриальной экономической системы для ускоренного темпа наращивания эфффекта ее функционирования Э.

Итак, в духе идей синергетики эволюция системы комбинирует фазы порядка и хаоса и протекает под знаком принципа «развитие через неустойчивость». В упорядоченной системе зарождается хаос, под влиянием которого в условиях сильной неравновесности она теряет устойчивость, и в точке бифуркации система может кардинально изменить характер своего развития, после чего в ней вновь воцаряется порядок. Подобные феномены самоорганизующейся системы формируют повестку дальнейших исследований - углубленного анализа и компьютерного моделирования сценариев потери и восстановления устойчивости развивающейся индустриальной экономической системы в нестационарной среде.

В завершение обобщим содержание статьи. Перед лицом угроз факторов возмущенной бизнес-среды российским промышленным предприятиям теоретическую и практическую значимость приобретает задача поддержания и повышения эффективной хозяйственной деятельности. В этих целях резонно обратиться к энтропийно-инфрормационной интерпретации и исследованию нетривиальных процессов, изучаемых теорией информации, синергетикой и смежными с ними отраслями знания.

Между тем эволюция индустриальной экономической системы в нестационарной среде с хаотическими и упорядоченными стадиями поведения подразумевает нелинейную динамику энтропии и испытывает воздействие возмущений и поступающей в систему управляющей информации. Насыщение системы этой информацией позволяет «изгонять» из нее хаос, повышать упорядоченность и эфрфект функционирования. Поэтому с познавательной и прикладной то- 
чек зрения для самоорганизации индустриальной экономической системы вызывают интерес поиск и анализ зависимости между показателями энтропии, накопленного количества информации и эфффекта фуункционирования системы на фроне дуализма в ней хаоса и порядка. На базе комплекса этих показателей в статье изложены результаты проведенного анализа, раскрывающие динамические свойства процесса самоорганизации индустриальной экономической системы.

\section{СПИСОК ИСПОЛЬЗОВАННОЙ ЛИТЕРАТУРЫ}

1. Второе начало термодинамики / С. Карно, У. Томсон (лорд Кельвин), Р. Клаузиус, Л. Больцман, М. Смолуховский ; под ред. А. К. Тимирязева. - 2-е изд. - М. : Изд-во ЛКИ, 2007. - 312 с.

2. Больцман Л. Статьи и речи / Л. Больцман. - М. : Наука, 1970. - 406 с.

3. Гиббс Дж. В. Термодинамика. Статистическая механика / Дж. В. Гиббс. - М. : Наука, 1982. - 584 с.

4. Шредингер Э. Что такое жизнь? С точки зрения физика : пер. с англ. / Э. Шредингер. - 2-е изд. - М. : Атомиздат, 1972. - 88 с.

5. Колмогоров А. Н. Избранные труды : в 6 т. / А. Н. Колмогоров. - М. : Наука, 2005. - Т. 3 : Теория информации и теория алгоритмов. -263 с.

6. Энциклопедия кибернетики : в 2 т. / под ред. В. М. Глушкова, Н. М. Амосова, И. А. Артеменко. Киев: Гл. ред. Укр. Сов. Энцикл., 1975. - Т. 1. - 608 с.

7. Винер Н. Кибернетика, или управление и связь в животном и машине : пер. с англ. / Н. Винер. 2-е изд. - М. : Наука, 1983. - 341 с.

8. Берталанфи Л., фон. Общая теория систем: критический обзор / Л. фон Берталанфи // Исследования по общей теории систем : пер. с англ. / под общ. ред. В. Н. Садовского, Э. Г. Юдина. - М. : Прогресс, 1969. - С. 23-82.

9. Бир С. Кибернетика и управление производством : пер. с англ. / С. Бир. - 2-е изд., доп. - М. : Наука, 1965. -392 c.

10. Бриллюэн Л. Научная неопределенность и информация : пер. с англ. / Л. Бриллюэн. - М. : Мир, 1966.- $271 \mathrm{c}$.

11. Шеннон К. Работы по теории информации и кибернетике : пер. с англ. / К. Шеннон. - М. : Изд-во иностр. лит., 1963. - 829 с.

12. Эшби У. Р. Введение в кибернетику : пер. с англ. / У. Р. Эшби. - М. : Изд-во иностр. лит., 1959. - 432 с.

13. Ферстер Г. О самоорганизующихся системах и их окружении / Г. Ферстер / / Самоорганизующиеся системы : пер. с англ. / под общ. ред. Т. Н. Соколова. - М. : Мир, 1964. - С. 113-139.

14. Экономика и менеджмент в условиях нелинейной динамики / А. А. Акимченко, А. А. Алетдинова, Е. М. Бухвальд, А. В. Бабкин, С. В. Чупров [и др.] ; под ред. А. В. Бабкина. - СПб. : Изд-во Политехн. ун-та, 2017. - 773 c.

15. Пригожин И. Порядок из хаоса: Новый диалог человека с природой : пер. с англ. / И. Пригожин, И. Стенгерс ; под общ. ред. В. И. Аршинова, Ю. Л. Климонтовича, Ю. В. Сачкова. - М. : КомКнига, 2005. - 296 с.

16. Трапезников В. А. Управление и научно-технический прогресс / В. А. Трапезников. - М. : Наука, 1983. -224 c.

\section{REFERENCES}

1. Carnot Sadi, Thomson William, $1^{\text {st }}$ Baron Kelvin, Clausius Rudolf, Boltzmann Ludwig, Smoluchowski Marian; Timiryazev A. K. (ed.). Vtoroe nachalo termodinamiki [The Second Law of Thermodynamics]. $2^{\text {nd }}$ ed. Moscow, LKI Publ., 2007. $312 \mathrm{p}$

2. Boltzmann Ludwig. Stat' i i rechi [Articles and Speeches]. Moscow, Nauka Publ., 1970. 406 p.

3. Gibbs Josiah Willard. Termodinamika. Statisticheskaya mekhanika [Thermodynamics. Statistical Mechanics]. Moscow, Nauka Publ., 1982. 584 p.

4. Sghrodinger Erwin. What is Life? The Physical Aspect of the Living Cell. Cambridge, The University Press, 1944. 91 p. (Russ. ed.: Sghrodinger Erwin. Chto takoe zhizn'? S tochki zreniya fizika. $2^{\text {nd }}$ ed. Moscow, Atomizdat Publ., 1972. 88 p.).

5. Kolmogorov A. N. Izbrannye trudy [Selected Works]. Moscow, Nauka Publ., 2005. Vol. 3. 263 p.

6. Glushkov V. M., Amosov N. M., Artemenko I. A. Entsiklopediya kibernetiki [Encyclopedia of Cybernetics]. Kiev, Ukrainian Soviet Encyclopedia Publ., 1974. Vol. 1. 608 p.

7. Wiener Norbert. Cybernetics or Control and Communication in the Animal and the Machine. Cambridge, Mass., The M.I.T. Press, 1948. (Russ. ed.: Wiener Norbert. Kibernetika, ili upravlenie i svyaz' v zhivotnom i mashine. $2^{\text {nd }}$ ed. Moscow, Nauka Publ., 1983. 341 p.).

8. Bertalanffy Ludwig von. General system theory - a critical review. In Sadovskii V. N., Yudin E. G. (eds). Issledovaniya po obshchei teorii sistem [Studies on the General Systems Theory]. Moscow, Progress Publ., 1969, pp. 23-82. (In Russian).

9. Beer Stafford. Cybernetics and Management. London, The English University Press, 1959. 214 p. (Russ. ed.: Beer Stafford. Kibernetika i upravlenie proizvodstvom. $2^{\text {nd }}$ ed. Moscow, Nauka Publ., 1965. 392 p.).

10. Brillouin Lon. Scientific Uncertainty \& Information. New York, Academic Press, 1964. 176 p. (Russ. ed.: Brillouin Lon. Nauchnaya neopredelennost' i informatsiya. Moscow, Mir Publ., 1966. 271 p.).

11. Shannon Claude. Raboty po teorii informatsii i kibernetike [Works on Information Theory and Cybernetics]. Moscow, Inostrannaya literatura Publ., 1963. 829 p. 
12. Ashby William Ross. An Introduction to Cybernetics. London, Chapman \& Hall, 1956. (Russ. ed.: Eshby William Ross. Vvedenie v kibernetiku. Moscow, Inostrannaya literatura Publ., 1959. 432 p.).

13. Foerster G. On Self-Organizing Systems and Their Environments. In Sokolov T. N. (ed.). Samoorganizuyushchiesya sistemy [Self-Organizing Systems]. Moscow, Mir Publ., 1964, pp. 113-139. (In Russian).

14. Akimchenko A. A., Aletdinova A. A., Bukhval'd E. M., Babkin A. V., Chuprov S. V. et al. Ekonomika $i$ menedzhment $v$ usloviyakh nelineinoi dinamiki [Economy and Management in Conditions of Non-Linear Dynamics]. Peter the Great Saint Petersburg Polytechnic University Publ., 2017. 773 p.

15. Prigogine llta, Istengers Isabelle. Order out of Chaos: Man's new dialogue with nature. London, Heinemann, 1984. 349 p. (Russ. ed.: Prigogine llya, Istengers Isabelle. Poryadok iz khaosa: Novyi dialog cheloveka s prirodoi. Moscow, KomKniga Publ., 2005. 296 p.).

16. Trapeznikov V. A. Upravlenie i nauchno-tekhnicheskii progress [Management and Technical Progress]. Moscow, Nauka Publ., 1983. 224 p.

\section{Информация об авторе}

Чупров Сергей Витальевич - доктор экономических наук, профессор, кафедра менеджмента, маркетинга и сервиса, Байкальский государственный университет, 664003, г. Иркутск, ул. Ленина, 11, e-mail: ChuprovSV@bgu.ru.

\section{Для цитирования}

Чупров С. В. Энтропийно-информационный анализ самоорганизации и эффректа функционирования индустриальной экономической системы / С. В. Чупров / / Известия Байкальского государственного университета. - 2017. - Т. 27, № 3. - С. 443-449. DOI: $10.17150 / 2500-2759.2017 .27(3) .443-449$.

\section{Author}

Sergey V. Chuprov - DSc in Economics, Professor, Department of Management, Marketing and Service, Baikal State University, 11 Lenin St., 664003, Irkutsk, Russian Federation, e-mail: ChuprovSV@bgu.ru.

\section{For Citation}

Chuprov S. V. Entropy-Information Analysis of Self-Organization and of the Effect of Functioning of an Industrial Economic System. Izvestiya Baykal'skogo gosudarstvennogo universiteta $=$ Bulletin of Baikal State University, 2017, vol. 27, no. 3, pp. 443-449. DOI: 10.17150/2500-2759.2017.27(3).443-449. (In Russian).

Редактор: Т. И. Кочульская. Переводчик: А. П. Бреева. Библиограф: В. М. Фомина. Верстка: А. С. Ларионова. Подписано в печать 11.09.17. Дата выхода 18.09.17. Формат $62 x 84$ 1/8. Тираж 1000 экз. Усл. печ. л. 18,0. Цена 550 р. Заказ 6570. Отпечатано в ИПО БГУ (664003, г. Иркутск, ул. Ленина, 11). 